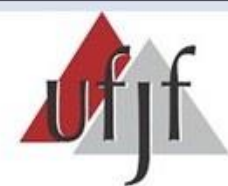

UNIVERSIDADE Federal de Juiz de Fora

\title{
ANOMALIAS DA CIRCULAÇÃO ATMOSFÉRICA ANTÁRTICA NA PRIMAVERA DE 2016 COMO INDUTORAS DE UMA CICLOGÊNESE EXPLOSIVA NO RIO GRANDE DO SUL
}

\section{ANTARTIC CIRCULATION ANOMALIES ON THE SPRING OF 2016 AS A INDUCTOR OF A EXPLOSIVE CYCLOGENESIS IN THE RIO GRANDE DO SUL}

\author{
VENISSE SCHOSSLER \\ Universidade Federal do Rio Grande do Sul \\ Av Bento Gonçalves, 9500, Agronomia, Porto Alegre- RS, CEP:90650-001
}

E-mail: venisse.schossler@ufrgs.br

FRANCISCO ELISEU AQUINO

Universidade Federal do Rio Grande do Sul Av Bento Gonçalves, 9500, Agronomia, Porto Alegre- RS, CEP:90650-001

E-mail: francisco.aquino@ufrgs.br

PEDRO AMARAL DOS REIS

Universidade Federal do Rio Grande do Sul Av Bento Gonçalves, 9500, Agronomia, Porto Alegre- RS, CEP:90650-001

E-mail:pamaralreis@gmail.com

JEFFERSON CARDIA SIMÕES

Universidade Federal do Rio Grande do Sul Av Bento Gonçalves, 9500, Agronomia, Porto Alegre- RS, CEP:90650-001

E-mail: jefferson.simoes@ufrgs.br

\section{Informações sobre o Artigo}

Data de Recebimento:

Novembro/2018

Data de Aprovação:

Dezembro/2018

\section{Resumo}

Este estudo examina a relação entre anomalias da circulação atmosférica antártica e uma ciclogênese explosiva na Bacia do Prata na primavera de 2016. A instabilidade se iniciou na Bacia do Prata, evoluindo para um ciclone extratropical que se deslocou para o Oceano Atlântico com fortes ventos sustentados gerando uma intensa maré de tempestade no litoral sul do Brasil. Para identificar a origem da ciclogênese, foram reconstruídos campos de altura geopotencial, vetores vento u e v e temperatura em 925, 500 e $200 \mathrm{hPa}$ da primavera e dos dias do evento (25 a 28/outubro) para o Hemisfério Sul com dados ERA/Interim. A associação entre o Modo Anular do Hemisfério Sul, padrão de onda três, ventos, altura e direção de ondas possibilitaram relacionar essa ciclogênese explosiva as anomalias de circulação atmosférica na região antártica.

Palavras-chave: variabilidade climática, aquecimento global, teleconexões climáticas, ENOS, SAM

\section{Abstract}

This study examines the relationship between Antarctic atmospheric circulation anomalies and an explosive cyclogenesis in the La Plata Basin in the 2016 spring. This instability began in the La Plata Basin, evolving into an extratropical cyclone, moved to the Atlantic Ocean with strong sustained winds generating an intense storm surge in the Brazilian southern coast. We reconstructed the fields of geopotential height, vectors wind $u$ and $v$ and temperature in 925,500 and $200 \mathrm{hPa}$ of the spring and the days of the event (25 to 28 / October) for the Southern Hemisphere with ERA / Interim data to identify the origin of the cyclogenesis. The association between the Southern Annular Mode, zonal wave three pattern, winds, height and wave direction made it possible to relate this explosive cyclogenesis to the atmospheric circulation anomalies in the Antarctic region.
\end{abstract}

Keywords: climate variability, global heat, climate teleconnections, ENSO, SAM 


\section{Introdução}

O ano de 2016 é, atualmente, o mais quente dos registros históricos (1880 a 2017) (NOAA, 2018). Na primavera de 2016 foi constatada a menor extensão de gelo marinho (SIE) desde o início da era satelital (TURNER et al., 2017). De acordo com Turner et al. (2017) os meses de setembro e outubro de 2016 apresentaram ondas planetárias amplificadas e um forte fluxo meridional, relacionados a uma série de tempestades nas latitudes médias. Schlosser et al. (2017) atribuem a rápida redução da SIE na primavera de 2016 a fase positiva do padrão de onda três (ZW3). A fase positiva do ZW3 intensifica os fluxos meridionais, impactando a SIE pelo transporte de ar quente/frio norte-sul/sul-norte (RAPHAEL, 2007). A intensificação do ZW3 foi atribuída por Stucker et al. (2017) as anomalias estacionárias da temperatura da superfície do mar (SST) do forte El Niño (EN) de 2015-2016 e a fase negativa do Modo Anular do Hemisfério Sul (SAM), forçante tropical sobre o Hemisfério Sul (HS), que nessa fase induz calor em direção à Antártica. Carvalho et al. (2005) notaram fortalecimento (enfraquecimento) dos jatos subtropicais de alto nível e enfraquecimento (fortalecimento) do jato polar de alto nível na fase negativa (positiva) do SAM. Assim, durante a fase negativa do SAM, a trajetória dos ciclones extratropicais é conduzida em direção ao equador junto aos jatos subtropicais, aumentando sua frequência em baixas latitudes (CARVALHO et al., 2005; REBOITA et al., 2009).

A baroclinia associada a variabilidade do SAM também influencia a ciclogênese e a storm track no HS (eg ALLAN e HAYLOCK 1993; RAO et al. 2003; SILVESTRE e VERA 2003, CARVALHO et al. 2005; GILLET et al. 2006; MENEGHINI et al. 2007; HENDON et al. 2007; REBOITA et al 2009; FIERRO e LESLIE 2013). Os ciclones migratórios são sistemas que influenciam a variabilidade climática e tendem a ter regiões preferenciais de formação e decadência. Estes sistemas desempenham um papel substancial no estoque de força e energia cinética, especialmente no HS (KEABLE et al. 2002). Devido a isso, a variabilidade espacial, sazonal e a intensificação dos ciclones extratropicais no HS tem recebido maior atenção nas últimas três décadas. Os ciclones do tipo explosivos, ou "bombas", são definidos como aqueles os quais a pressão central diminui rapidamente, em média 1 hectorpascal ( $\mathrm{hPa}$ ) por hora em 24 horas, com rápido aumento da velocidade dos ventos geostróficos, refletindo ventos mais fortes na superfície (SANDERS e GYAKUM 1980; WATSON et al. 2000). Essa categoria de sistema de baixa pressão tem diminuído nas latitudes médias e aumentado nas altas latitudes desde a década de 1970 em ambos hemisférios (ALLEN et al. 2010). O inverno é o período de maior ocorrência de ciclones explosivos globalmente, embora a frequência dos ciclones explosivos no HS não possua a marcada sazonalidade do HN (GAN e RAO 1991; ALLEN et al. 2010). Quanto a distribuição espacial dos ciclones explosivos no HS, Allen et al. (2010) aplicaram múltiplas reanálises para identificar as áreas preferencias. A maior densidade se encontra em uma larga 
faixa que se estende do Oceano Atlântico à oeste da América do Sul (entre $70^{\circ}$ e $10^{\circ} \mathrm{W} ; 30^{\circ} \mathrm{e}$ $\left.50^{\circ} \mathrm{S}\right)$.

Na primavera de 2016, durante a fase negativa do SAM, positiva do ZW3 e SST anômala no Oceano Austral, foi também identificada ciclogênese anômala na região subtropical do HS. Entre os dias 26 e 28 de outubro de 2016, um intenso ciclone extratropical se formou a partir de uma instabilidade na Bacia do Prata, especialmente entre o Uruguai e o Rio Grande do Sul (RS), se deslocando para o sudoeste do Atlântico Sul. Este ciclone gerou fortes rajadas de vento que atingiram a costa sul do Brasil, provocando maré de tempestade e, consequentemente, inundações ao longo de $605 \mathrm{~km}$ de costa. Casas foram destruídas e aproximadamente 145 mil pessoas ficaram sem energia. Logo, com a intensão de relacionar a ciclogênese anômala no HS no final de outubro de 2016 com as anomalias atmosféricas e oceânicas na Antártica na primavera de 2016, foram examinadas as anomalias geopotenciais dos campos de pressão, temperatura e vento ( $u$ e v) no HS, índice do ZW3 e dados meteorológicos das áreas atingidas, contribuindo para a compreensão da relação entre as anomalias da circulação atmosférica nas regiões polares e eventos meteorológicos nas regiões extratropicais.

\section{Metodologia}

Neste trabalho foram utilizados os bancos de dados Dayli e Monthly Means of Dayli Means da reanálise atmosférica ERA-Interim da ECMWF (European Centre for Medium-Range Weather Forecasts). Os dados Dayli cobrem o período do dia 25 ao dia 31 de outubro de 2016, com resolução horizontal de $0.1^{\circ}$ x $0.1^{\circ}$ (latitude/longitude), os dados de Monthly Means of Dayli Means utilizados são da primavera austral 2016, com resolução horizontal de $0.5^{\circ} \times 0.5^{\circ}$. Os dados utilizados foram altura geopotencial em $500 \mathrm{hPa}$, temperatura do ar em $925 \mathrm{hPa}$, vento meridional em $925 \mathrm{hPa}$ e vento zonal em $200 \mathrm{hPa}$. A partir desses dados foram calculadas as anomalias da primavera austral e do mês de outubro com base na climatologia 1979-2016. Já as diárias do mês de outubro foram calculadas com base no próprio mês.

O ZW3 Index foi calculado para primavera de 2016, segundo Raphael (2004), e o resultado

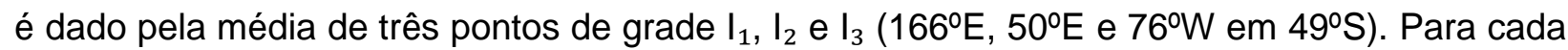
ponto é utilizado a equação 1 :

$$
I_{i}=\frac{\left(X_{m t h l y}-X_{m t h l y}\right)}{\sigma_{m t h l y}}
$$

Onde $X_{m t h l y}$ é a média de três meses, $X_{m t h l y}$ é a média climatológica dos três meses e $\sigma_{m t h l y}$ é o desvio padrão da média dos três meses. Dessa forma, valores positivos no índice 
indicam fortalecimento dos ventos meridionais e valores negativos indicam predominância do vento zonal. Os dados utilizados para o cálculo foram do ERA Interim, com climatologia de 19792016. São esperados resultados diferentes para o ZW3 Index em comparação com os Raphael (2007) que utilizou outra climatologia (1958-2005) e banco de dados (NCEP-NCAR I reanalysis).

O índice SAM utilizado é o de Marshall (2003), calculado pela diferença de pressão zonal entre as latitudes de $40^{\circ}$ e $65^{\circ} \mathrm{S}$.

Para interpretar as relações entre as anomalias Antárticas e a maré de tempestade durante o evento, foram utilizados dados de altura de ondas, direção das ondas e ventos da boia Rio Grande (3131' 12.0" S, 49 48' 36.0” W) (http://www.goosbrasil.org/pnboia/dados/) do Programa Nacional de Boias (PNBOIA) do Brasil. As medições são automáticas e de hora em hora.

A classificação de ciclones explosivos adotada foi a de Sanders e Gyakum (1980). Ciclones explosivos são aqueles os quais a pressão diminui em torno de $24 \mathrm{hPa}$ em 24 horas, na latitude de $60^{\circ}$. Para outras latitudes, o critério é multiplicar por uma correção através da Taxa Normalizada de Aprofundamento da pressão central (NDRc), apresentada na equação (2):

$$
N D R_{c}=\left(\frac{\sin 60^{\circ}}{\sin \emptyset}\right) \cdot\left(\frac{\Delta p_{c}}{24 h P a}\right)
$$

onde, $\Delta \mathrm{pc}$ é a diferença de pressão no sistema, em 24 horas e $\Phi$ é a latitude média de localização do centro. Uma taxa de profundidade de $24 \mathrm{hPa}$ em 24 horas, multiplicado por (sin $60^{\circ} / \sin \emptyset$ ) é definido como 1 bergeron, da latitude de Berger, $60^{\circ}$.

\section{Resultados e Discussões}

Como já citado, a circulação atmosférica antártica na primavera de 2016 foi caracterizada por anomalias, induzidas por marcado ZW3 e altas temperaturas no Oceano Austral (SCHLOSSER et al., 2017; STUCKER et al., 2017). Além disso, os meses de outubro e novembro apresentaram SAM negativo, que, caracteristicamente, induz calor dos trópicos para Antártica e frio da Antártica para os trópicos (HALL e VISBECK, 2001).

Na Figura 1 é possível identificar os campos das anomalias geopotencial em 500 hPa nos dias 27 e 28 de outubro (dias de intensificação do evento), que deixam nítido o ZW3, com anomalias negativas no Oceano Pacífico/Mar de Amundsen e nos setores oeste e leste do Oceano Índico Sul, e anomalias positivas no Oceano Pacífico/mares de Ross e Bellinghausen, e setor central do Oceano Índico Sul. No mesmo período, é possível observar na Figura 2 as anomalias dos campos de ventos zonal em $200 \mathrm{hPa}$, com fraco fluxo zonal sobre o Oceano 
Austral e forte nas latitudes médias, característicos de períodos de SAM negativo, ainda que fossem um pouco mais intensos sobre a Península Antártica e o Mar de Amundsen e menos intensos sobre o sul da América do Sul e Oceano Atlântico. Na Figura 2 também é possível identificar anomalias negativas nos ventos de oeste em $200 \mathrm{hPa}$.

No mês de outubro, a circulação atmosférica do HS esteve sob domínio do SAM negativo (-0.89). Novembro o SAM permaneceu negativo (-3.12), com um incremento nas anomalias negativas do vento zonal em $200 \mathrm{hPa}$, assim como, o domínio das anomalias geopotencial em 500 hPa sobre a região Antártica. Hall e Visbeck (2001) caracterizam as implicações do SAM no sistema climático do HS. Os autores afirmam que quando o SAM é negativo (positivo) ocorre diminuição (aumento) do gelo marinho, associada ao enfraquecimento (intensificação) dos ventos de oeste em superfície, e aumento (diminuição) da SST. Assim, o comportamento do campo de pressão e circulação atmosférica no HS induziram a organização de ondas planetárias amplificadas, com forte fluxo meridional, característicos de ZW3. O índice de ZW3 calculado teve resultado de +0.56 para a combinação dos meses de setembro, outubro e novembro (SON) de 2016. Esse valor é anomalamente positivo em relação à média histórica 1958-2001 para SON calculados por Raphael (2004), que tem valor aproximado de -0.60. Este ZW3 foi, e é, responsável pela conexão trópico-polo/polo-trópico gerando eventos meteorológicos na escala sinótica. A fase positiva do ZW3 intensifica os fluxos meridionais, impactando a SIE pelo transporte de ar quente/frio norte-sul/sul-norte (RAPHAEL, 2007). Estas condições foram as mesmas descritas por Turner et al. (2017), como as responsáveis pelo recorde de retração de gelo marinho ao redor da Antártica e tempestades ciclônicas nas latitudes médias na primavera de 2016.

Essas condições atmosféricas também favoreceram o evento meteorológico nos últimos dias do mês de outubro de 2016 no litoral do RS. Nos dias 27 e 28 de outubro de 2016 foram registrados fortes ventos e maré de tempestade. Na verdade, toda costa sul e sudeste do Brasil foi atingida, porém, pela proximidade do ciclone, o sul do RS foi o mais atingido. No Balneário do Hermenegildo (3339'59'S; 5315'39'W) a população ficou sem água e luz por 4 dias, 53 casas foram destruídas e 20 danificadas. No total foram 50 mil pessoas sem energia elétrica no Estado. $\mathrm{Na}$ cidade portuária de Rio Grande (3202'12'S; $52^{\circ} 06^{\prime} 22^{\prime \prime} \mathrm{W}$ ), as escolas e o porto foram fechados e tiveram todas atividades suspensas por 2 dias devido a rajadas de vento de até 31 $\mathrm{m} \mathrm{s}^{-1}$. A costa do RS é governada por ondas de $1,5 \mathrm{~m}$ em média, com regime de micromaré $(0,3$ $\mathrm{m})$ também condicionado por correntes longitudinais. Os ventos predominantes são de nordeste, mas os de sudoeste são os mais competentes e intensos (CALLIARI e KLEIN, 1992; TOMAZELLI, 1993). De acordo com a classificação climática de Strahler (1989), o RS tem clima controlado por massas de ar tropicais e polares, situado em uma região subtropical, de transição entre baixas e médias latitudes. Nessa localização, o estado é preferencialmente influenciado por ciclones e sistemas frontais no inverno e sistemas convectivos no verão. 

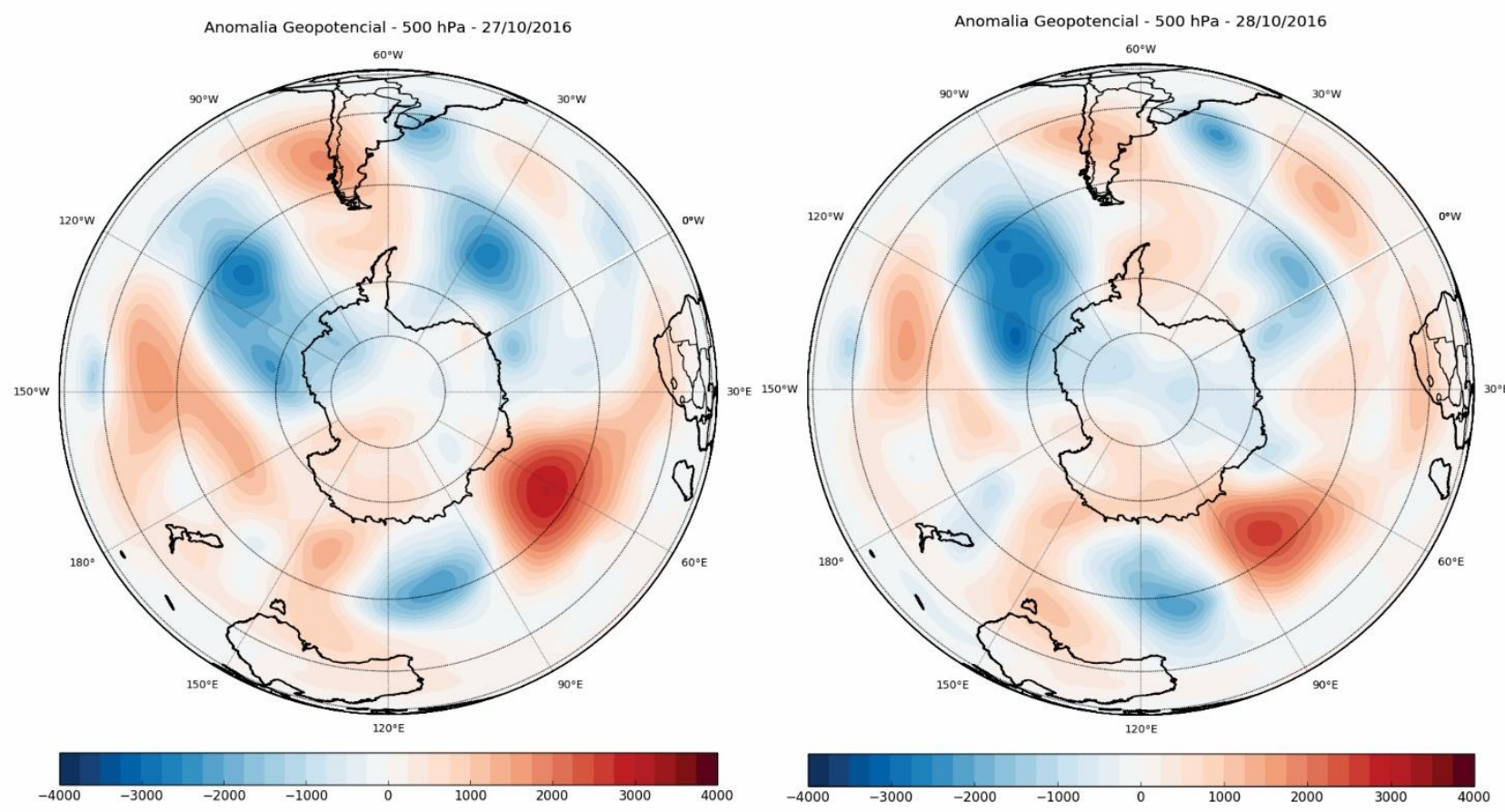

FIGURA 1: Mapas de anomalias de altura geopotencial em 500 hPa no Hemisfério Sul, nos dias 27 e 28 de outubro de 2016.
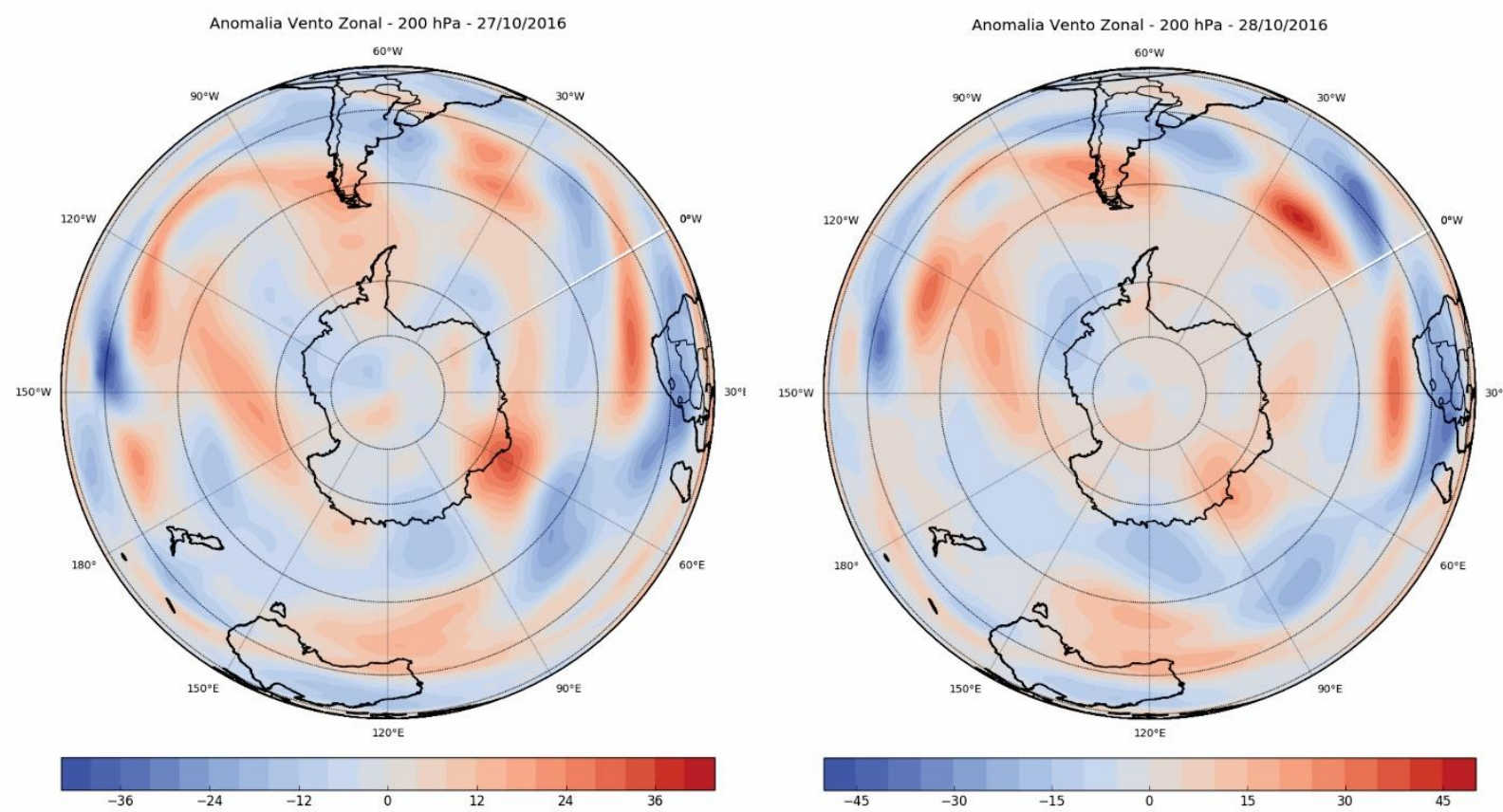

FIGURA 2: Mapas de anomalias de vento zonal em 200 hPa no Hemisfério Sul nos dias 27 e 28 de outubro de 2016.

Ao observar a Tabela 1 é possível perceber o desenvolvimento da maré de tempestade durante o evento meteorológico. A evolução da velocidade do vento e rajadas chegou ao seu pico na cidade de Rio Grande na tarde do dia 27. Com rajadas de até $28 \mathrm{~m} \mathrm{~s}^{-1}$ de direções SSW, as ondas se aproximaram da costa com altura 3 vezes superior que a média, de direção predominante SSW/ESW. Junto com a maré de tempestade formada, a temperatura caiu 5ㄷ $\mathrm{e}$ 
a pressão atmosférica baixou para $1000 \mathrm{hPa}$ na superfície. Durante a tempestade, a boia que media as marés se perdeu, impossibilitando a medida. Nas cartas sinóticas de superfície e altitude dos dias do evento (Figura 3) é possível identificar a evolução da instabilidade para uma ciclogênese sobre a Bacia do Prata e seu deslocamento e intensificação sobre o Atlântico Sul.

TABELA 1

Variáveis meteorológicas em Rio Grande, Rio Grande do Sul, no evento de outubro de 2016.

\begin{tabular}{c|c|c|c|c|c|c|c}
\hline Data & $\begin{array}{c}\text { Vento } \\
(\mathrm{m} / \mathrm{s})\end{array}$ & $\begin{array}{c}\text { Rajadas } \\
(\mathrm{m} / \mathrm{s})\end{array}$ & $\begin{array}{c}\text { Direção } \\
\text { do vento }\end{array}$ & $\begin{array}{c}\text { Pressão } \\
(\mathrm{hPa})\end{array}$ & $\begin{array}{c}\text { Altura } \\
\text { significativa } \\
(\mathrm{m})\end{array}$ & $\begin{array}{c}\text { Direção } \\
\text { da onda }\end{array}$ & $\begin{array}{c}\text { Temperatura } \\
{ }^{\circ} \mathrm{C}\end{array}$ \\
\hline $25 / 10$ & 10,28 & 16 & $170^{\circ}$ & 1017 & 1,94 & $132^{\circ}$ & 17 \\
\hline $26 / 10$ & 5,14 & 15 & $150^{\circ}$ & 1011 & 1,83 & $105^{\circ}$ & 19 \\
\hline $26 / 10$ & 5,14 & 12 & $150^{\circ}$ & 1003 & 1,24 & $90^{\circ}$ & 19 \\
\hline $\mathbf{2 7 / 1 0}$ & $\mathbf{9 , 7 7}$ & $\mathbf{1 6}$ & $\mathbf{3 5 0 ^ { \circ }}$ & $\mathbf{9 9 8}$ & $\mathbf{1 , 9 3}$ & $\mathbf{2 5 5 ^ { \circ }}$ & $\mathbf{1 3}$ \\
\hline $\mathbf{2 7 / 1 0}$ & $\mathbf{1 3 , 3 7}$ & $\mathbf{2 8}$ & $\mathbf{2 0 0 ^ { \circ }}$ & $\mathbf{9 9 5}$ & $\mathbf{3 , 5}$ & $\mathbf{2 3 7 ^ { \circ }}$ & $\mathbf{1 2}$ \\
\hline $\mathbf{2 8} / \mathbf{1 0}$ & $\mathbf{0 , 5 1}$ & $\mathbf{2 3}$ & $\mathbf{4 0}$ & $\mathbf{1 0 0 8}$ & $\mathbf{5 , 1 6}$ & $\mathbf{2 2 5 ^ { \circ }}$ & $\mathbf{1 8}$ \\
\hline $\mathbf{2 8} / \mathbf{1 0}$ & $\mathbf{9 , 2 5}$ & $\mathbf{1 8}$ & $\mathbf{2 2 0 ^ { \circ }}$ & $\mathbf{1 0 1 8}$ & $\mathbf{4 , 9 4}$ & $\mathbf{2 0 1 ^ { \circ }}$ & $\mathbf{1 5}$ \\
\hline $29 / 10$ & 3,08 & 5 & $180^{\circ}$ & 1026 & 3,81 & $195^{\circ}$ & 16 \\
\hline
\end{tabular}

Fonte: PROGRAMA NACIONAL DE BOIAS DO BRASIL, 2017.
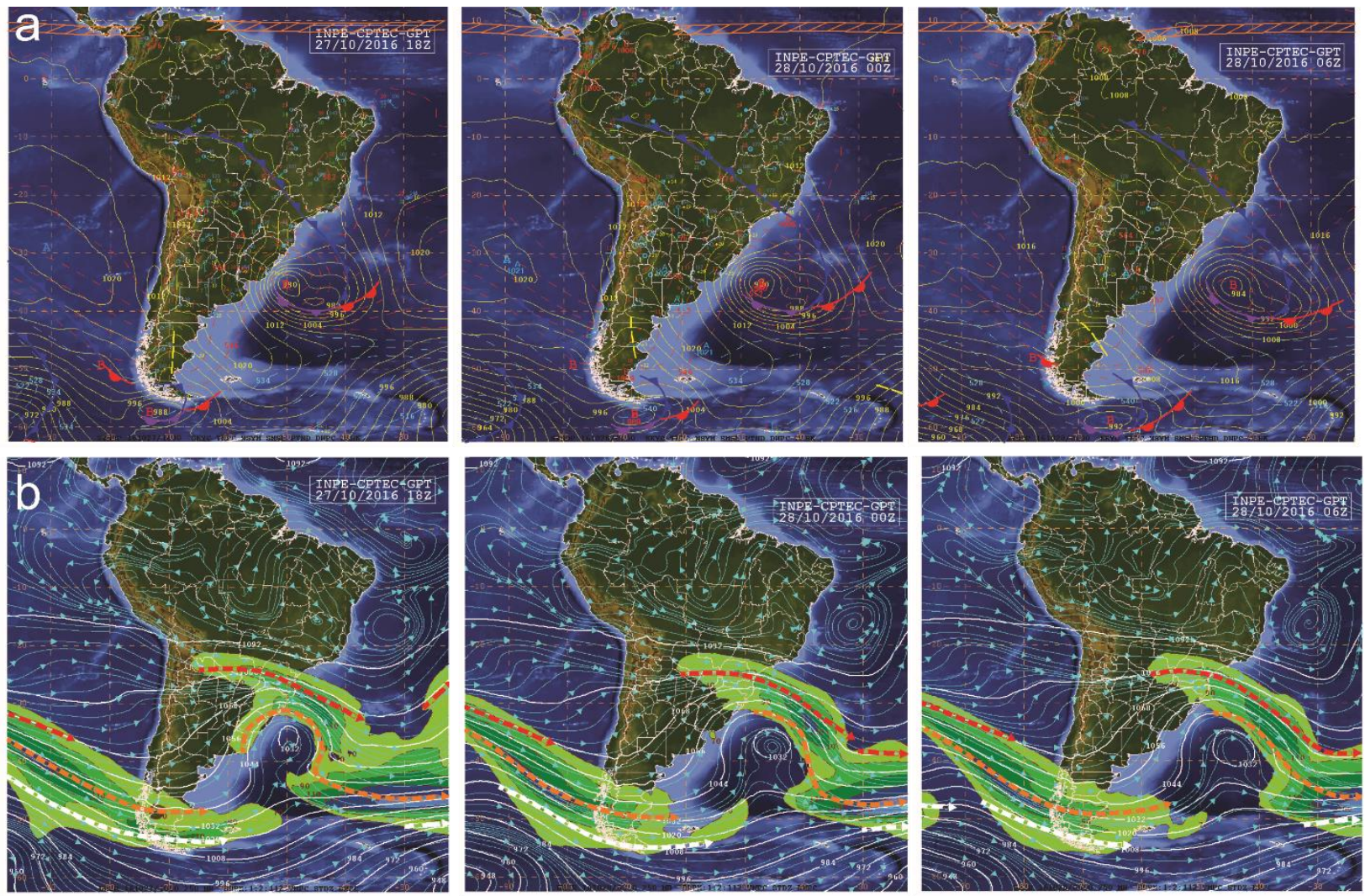

FIGURA 3: Cartas sinóticas do dia 27 às 18 UTC e 28 de outubro da 00 e 06 UTC, momento de intensificação da ciclogênese e oclusão do ciclone extratropical. Em a) as cartas em superfície e em b) cartas em altitude. Fonte: CPTEC, 2017.

A ciclogênese originada a partir da instabilidade no dia 27 pode ser caracterizada como ciclone do tipo explosivo, pois registrou-se em seu centro $1006 \mathrm{hPa}$ as 18 UTC do dia 26, 
diminuindo para $986 \mathrm{hPa}$ as 18 UTC do dia 27. De acordo com Sanders e Gyakum (1980), um ciclone do tipo explosivo, ou "bomba", é definido como um ciclone extratropical em superfície o qual sua pressão central diminui até $1 \mathrm{hPa}$ /hora em 24 horas na latitude de $60^{\circ}$, para outras latitudes aplica-se uma variável de normalização. A queda de pressão foi de $0,8 \mathrm{hPa}$ por hora durante 24 horas (que na latitude da ocorrência é considerado explosivo), com circulação de sul induzida pela ciclogênese e queda de temperatura, registrados entre 9 e $5^{\circ} \mathrm{C}$. No dia 28 o ciclone atuava ao longo da costa do sul do Brasil e do Uruguai com pressão central inferior a $984 \mathrm{hPa}$, temperaturas entre 3 e $10^{\circ} \mathrm{C}$ e ventos ainda do quadrante sul (Figura $4 \mathrm{a}$ e $4 \mathrm{~b}$ ). $\mathrm{O}$ avanço de um anticiclone migratório polar (1024 hPa) sobre a Bacia do Prata no dia 29 deslocou o ciclone para leste, diminuindo a intensidade dos ventos na costa sul do Brasil, ainda que continuassem oriundos do quadrante sul. 
Anomalia Geopotencial - $500 \mathrm{hPa}$ - 27/10/2016
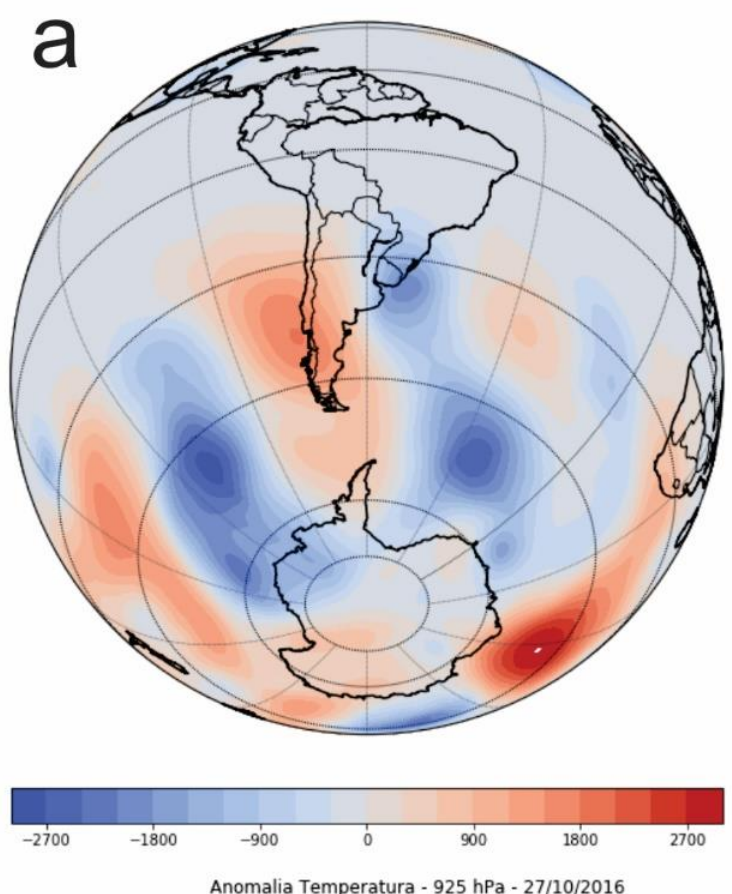

b

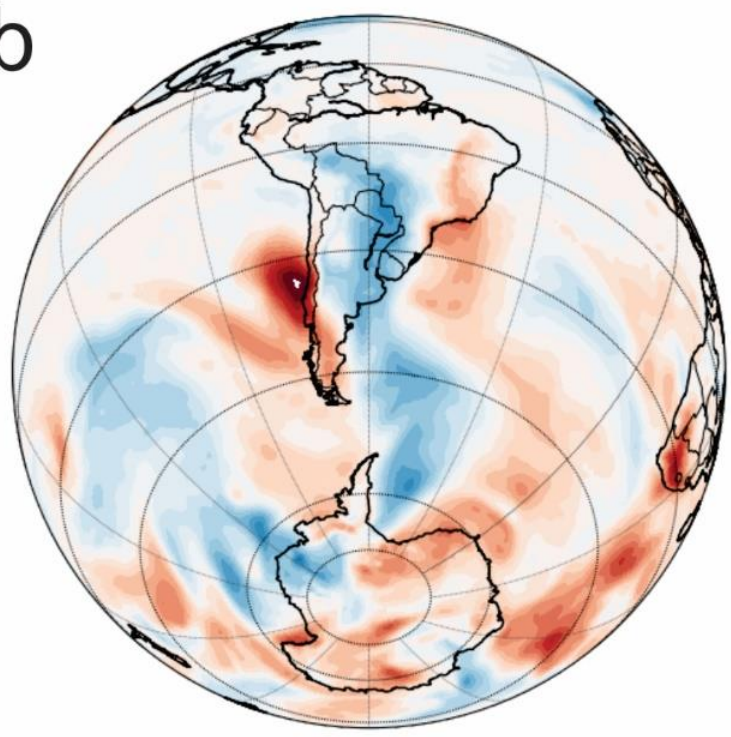

Anomalia Geopotencial - $500 \mathrm{hPa}-28 / 10 / 2016$
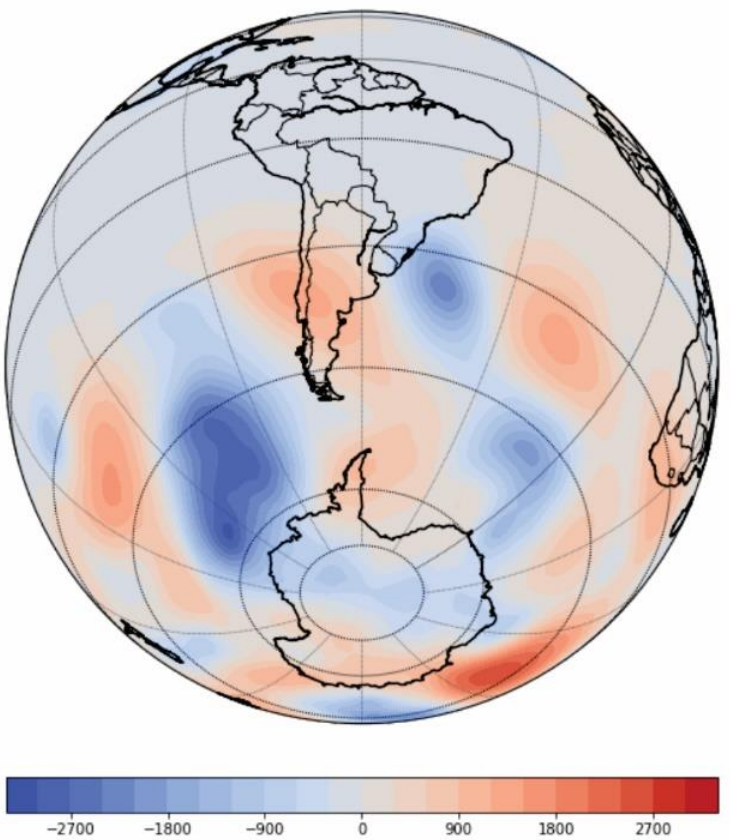

Anomalia Temperatura - $925 \mathrm{hPa}-28 / 10 / 2016$
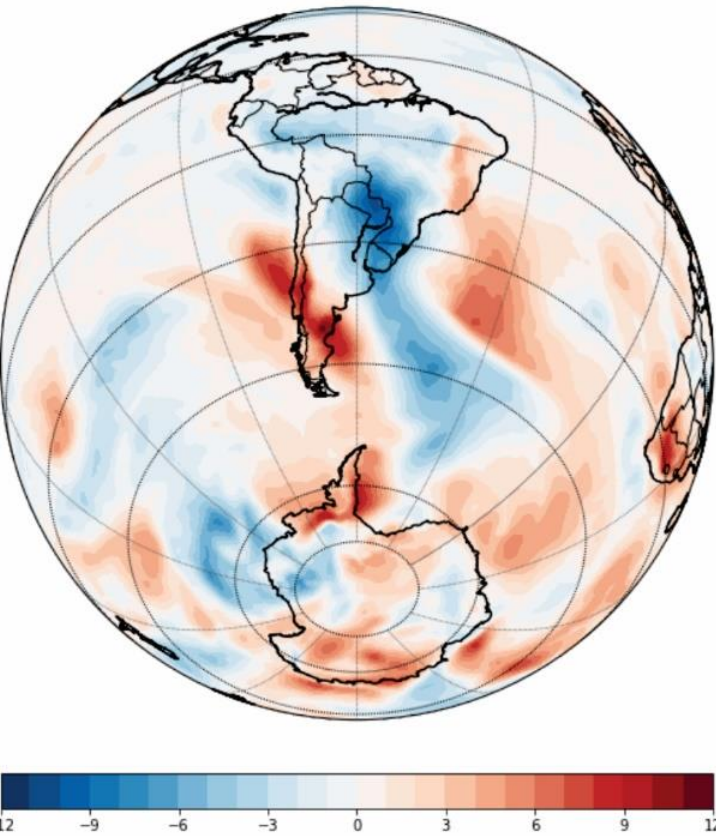

FIGURA 4: Mapas de anomalias de altura geopotencial em $500 \mathrm{hPa}$ (a) e, temperaturas em $925 \mathrm{hPa}$ (b) entre a Antártica e sul do Brasil nos dias 27 e 28 de outubro de 2016.

\section{Considerações Finais}

A área de instabilidade que se formou na Bacia do Prata no dia 25 de outubro de 2016 evoluiu para um ciclone explosivo nos dias 27 e 28 enquanto se deslocava para leste, setor oeste do Atlântico Sul. Essa ciclogênese explosiva possui clara relação sinótica com a organização do intenso ZW3 no HS durante a primavera de 2016. Esse ZW3 anômalo foi responsável pelo recorde de retração do gelo marinho ao redor da Antártica e por uma série tempestades nas 
latitudes médias no mesmo período. Associado ao marcado ZW3, foi registrado SAM negativo, que também intensifica a ciclogênese nas regiões subtropicais, ao mesmo tempo em que enfraquece os ventos de oeste em latitudes médias e amplifica a troca de frio/calor entre as regiões polares e tropicais.

A maré de tempestade registrada no litoral do RS foi resultante dos intensos ventos e ondas do quadrante SSW, governados pelo ciclone explosivo que se posicionava a SSE, ao largo da costa do Estado. De acordo com as cartas sinóticas e campos de anomalias de geopotencial 500 hPa e de temperatura em superfície é possível observar o ar frio oriundo da região antártica, que provocou queda nas temperaturas mínimas e médias em todo sul do Brasil nos últimos dias de outubro de 2016.

Portanto, os resultados aqui encontrados contribuem para a compreensão de como anomalias atmosféricas no HS podem ser controladas pela região antártica e se teleconectam com as regiões subtropicais/tropicais, em especial a região Sul do Brasil. O aquecimento global vem intensificando a ocorrência de anomalias climáticas no planeta e, em especial, nas regiões polares, alertando para a importância de se entender as relações/teleconexões climáticas dessas regiões com as regiões tropicais e subtropicais, onde vive grande parte da população terrestre.

\section{Agradecimentos}

Os autores agradecem à Fundação de Amparo à Pesquisa do Estado do Rio Grande do Sul (FAPERGS) e ao Instituto Nacional de Ciência e Tecnologia da Criosfera (INCT da Criosfera) pelo apoio financeiro e ao NOTOS - Laboratório de Climatologia pelo suporte acadêmico.

\section{Referências Bibliográficas}

ALLAN, R. J.; HAYLOCK M. R. Circulation Features Associated with the Winter Rainfall Decrease in Southern Australia. Journal of Climate https://doi.org/10.1175/15200442(1993)006\%3C1356:CFAWTW\%3E2.0.CO;2. 1993.

ALLEN, J. T., PEZZA, A. B.; BLACK, M. T. Explosive Cyclogenesys: A Global Climatology Comparing Multiple Reanalyses. Journal of Climate. https://doi.org/10.1175/2010JCLI3437.1 . 2010.

CALLIARI, L. J.; KLEIN, A. H. F. Características morfodinâmicas e sedimentológicas das praias oceânicas entre Rio Grande e Chuí, RS. In: CONGRESSO BRASILEIRO DE GEOLOGIA, 37, 1992, São Paulo, Abstracts Volume, São Paulo, 1992. p. 77-78.

CARVALHO, L. M. V.; JONES, C.; AMBRIZZI, T. Opposite Phases of Antartic Oscillation and the relationships with intraseasonal to interannual activity in the tropics during the austral summer. Journal of Climate, v. 18, p.702-718. http://doi/pdf/10.1175/JCLI3284. 2005.

CPTEC, Centro de Previsão do Tempo e Estudos Climáticos. Análise Sinótica. http://tempo.cptec.inpe.br/ , acesso em 30 de novembro de 2017.

ECMWF, European Centre for Medium-Range Weather Forecasts, Climate Reanalysis, https://www.ecmwf.int/en/research/climate-reanalysis, acessado em 18 de dezembro de 2017.

FIERRO, A. O; LESLIE, L. M. Links between Central West Western Australian Raifall Variability and LargeScale Climate Drivers. Journal of Climate. https://doi.org/10.1175/JCLI-D-12-00129.1 .2013. 
GAN, M. A.; RAO, V. B. Surface Cyclogenesis over South America. Monthly Weather Review. https://doi.org/10.1175/1520-0493(1991)119\%3C1293:SCOSA\%3E2.0.CO;2. 1991.

GILLET, N. P; KELL, T. D.; JONES, P.D. Regional climate impacts of the Southern Annular Mode. Geophysical Research Letters. https://doi.org/10.1029/2006GL027721. 2006.

HALL, A.; VISBECK, M. Ocean and Sea Ice response to the Southern Hemisphere Annular Mode: Results from a coupled climate model. Clivar Exchanges, n. 22, 4 p. 2001.

HENDON, H. H.; LIM, E.; NGUYEN, H. Seasonal Variations of Subtropical Precipitation Associated with the Southern Annular Mode. Journal of Climate. https://doi.org/10.1175/JCLI-D-13-00550.1. 2014.

KEABLE, M.; SIMMONDS, I.; KEAY, K. (2002) Distribution and Temporal Variability of $500 \mathrm{hPa}$ Cyclone Characteristics in the Southern Hemisphere. International Journal of Climatology. https://doi.org/10.1002/joc.728. 2002.

MARSHALL, G. J. Trends in the Southern Annular Mode from observations and reanalyzes. Journal of Climate, n. 16, p. 4134-4143. 2003.

MENEGHINI, B.; SIMMONDS, I.; SMITH, I. N. Association between Australian rainfall and the Southern Hemisphere. International Journal of Climatology. https://doi.org/10.1002/joc.1370. 2007.

NOAA, National Oceanic and Atmospheric Administration. Climate Prediction Center. http://www.cpc.ncep.noaa.gov/data/teledoc/teleintro.shtml , acesso em 22 de abril de 2018.

PNBOIA, Programa Nacional de Boias, http://www.goosbrasil.org/pnboia/dados, acessado em 30 de novembro de 2017.

RAO V. B.; CARMO, A. M. C.; FRANCHITO, S. H. Interannual variations of storm tracks in the Southern Hemisphere and their connections with the Antartic Oscillation. International Journal of Climatology, http://doi.10.1002/joc.948. 2003.

RAPHAEL, M. N. A zonal wave 3 index for the Southern Hemisphere. Geophysical Research Letters, v. 31, L23212, 4 p, http://doi:10.1029/2004GL020365 . 2004.

RAPHAEL, M. N. The influence of atmospheric zonal wave three on Antarctic sea ice variability. Geophysical Research Letters, v. 112, D12112, 9 p, http://doi:10.1029/2006JD0078522007 . 2007.

REBOITA, M.S., AMBRIZZI, T., DA ROCHA, R.P. Relationship between Southern Annular Mode and Southern Hemisphere atmospheric systems. Revista Brasileira de Meteorologia v .1, p. 48-55. 2009.

SANDERS, F.; GYAKUM, J. R. Synoptic-Dynamic Climatology of the "Bomb". Monthly Weather Review, n. 108, p. $1589-1606.1980$.

SCHLOSSER, E.; HAUMANN, F. A.; RAPHAEL, M. N. Atmospheric influences on the anomalous 2016 Antarctic sea ice decay. The Cryosphere Discuss, n. 12, p. 1103-1119. https://doi.org/10.5194/tc-121103-2018. 2018.

SILVESTRI, G. E.; VERA, C. S. Antartic Oscillation signal on precipitation anomalies over southeastern South America. Geophysical Research Letters. https://doi.org/10.1029/2003GL018277. 2003.

STRAHLER, A. N.; STRAHLER, A. H. Geografia Física. Barcelona: Ediciones Omega. 1989.

STUCKER, M. F.; BITZ, C. M.; ARMOUR, K.C. Conditions leading to the unprecedent low Antarctic sea ice extent during the 2016 austral spring season. Geophysical Research Letters, n. 44, p. 9008-9019, http://doi:10.1002/2017GL074691 . 2017.

TOMAZELLI, L. J. O regime de ventos e a taxa de migração de dunas eólicas costeiras do Rio Grande do Sul, Brasil. Pesquisas em Geociências, v. 20 (1), p. 18-26. 1993.

TURNER, J.; PHILLIPS, T.; MARSHALL, G. J.; HOSKING, S.; JAMES O. POPE, J. O.; BRACEGIRDLE, T. J.; DEB, P. Unprecedented springtime retreat of Antarctic sea ice in 2016. Geophysical Research Letters, v. 44, p. 6868-6875. 2017.

WATSON, G.; HALLIDAY, J. A.; PALUTIKOF, J. P.; HOLT, T.; BARTHELMIE, R. J.; COELINGH, J. P.; FOLERTS, L.; VAN ZUYLEN, E. J.; CLEIJNE, J. W. POWER: A methodology for predicting offshore wind energy resources. Proc. European Seminar on Offshore Wind Energy in the Mediterranean and other European Seas (OWEMES). Siracusa, Sicily, Italy, European Wind Association, 10 p. 2000. 